\title{
Sparsity-aware 3D ToF Sensing
}

This paper was downloaded from TechRxiv (https://www.techrxiv.org).

\section{LICENSE}

CC BY 4.0

SUBMISSION DATE / POSTED DATE

14-02-2022 / 22-02-2022

CITATION

Lopez Paredes, Alvaro; Loffeld, Otmar; Heredia Conde, Miguel (2022): Sparsity-aware 3D ToF Sensing. TechRxiv. Preprint. https://doi.org/10.36227/techrxiv.19161749.v1

$\mathrm{DOI}$

10.36227/techrxiv.19161749.v1 


\title{
Sparsity-aware 3D ToF Sensing
}

\author{
Alvaro Lopez Paredes, Miguel Heredia Conde, and Otmar Loffeld
}

\begin{abstract}
In this work, several ToF sensing schemes which tackle the challenge of obtaining high angular resolution and long ranges in nearly real-time, with relatively simple implementation and low associated computational load, are proposed. Sliced Orthogonal Matching Pursuit (OMP) divides the spatial domain in a number of partitions which ensure an efficient projection of the scene by optimizing the inter-column coherence of the sensing matrices. The signals are preliminarily localized within the partitions and OMP is then applied to recover depth and amplitude in a refined spatial domain. The preliminary set of measurements reduces the effective domain of the signal, lowers the processing times, and improves the sensing accuracy. Several methodologies are described for the construction of the sensing matrices, such as Low-Density Parity-Check codes via Progressive Edge Growth (LDPC-PEG), and random permutations of $(0,1)$-binary columns generated as combinations without repetition of a fixed number of nonzero elements for each column. Furthermore, we extend the previous schemes accounting for the raising and falling edges in order to avoid any possible coincidence which may degrade the coherence. Then, the upper super-resolution limit is studied accounting for the Instrument Response Function (IRF) of the ToF sensor. Zoned APEG extends the applicability of Adaptive Progressive Edge Algorithm (APEG) to more practical illumination systems by considering several groups of signals, arising from different areas of the sensor array, during the adaptation of the sensing matrices. The signals are then individually retrieved for each pixel via OMP over the identified joint signal support.
\end{abstract}

Index Terms-Adaptive compressive sensing, imaging sensors, sparsity-awareness, Time-of-Flight.

\section{INTRODUCTION}

Over the past few years, Compressive Sensing (CS) theory has attained a prominent position within the computer vision and signal processing communities. The early works of Candès [1]-[3] and Donoho [4] demonstrated that a periodic signal can be recovered without prior knowledge by fewer measurements than the lower bounds previously established by the Whittaker-Kotel'nikov-Shannon (WKS) sampling theorem if represented in an appropriate basis. Since then, numerous efforts have been invested to push these limits further. 3D imaging, and specially ToF systems, are one of the fields where the echoes of CS techniques have more actively resounded. ToF imaging systems implicitly aim to find the support of an ideally-one-sparse signal in depth domain (for single targets) by means of a $2 \mathrm{D}$ pixel array by calculating the time, i.e., distance, required by the light to travel from the sensor to the scene under study and back. In Pulse-based ToF (PBToF) systems, the sensors can be seen as correlators between a delayed light pulse and a number of binary sequences, randomly or deterministically generated [5], [6]. The delay

This project has received funding from the European Union's Horizon 2020 research and innovation programme under the Marie SkłodowskaCurie grant agreement No 860370 . The contribution of the first author is part of a Ph.D. thesis research conducted at the University of Siegen.

Alvaro Lopez Paredes, Miguel Heredia Conde, and Otmar Loffeld are with the Center for Sensor Systems (ZESS) of the University of Siegen, 57076 Germany (e-mail: alvaro.Iparedes@uni-siegen.de, heredia@zess.uni-siegen.de, loffeld@zess.uni-siegen.de

A version of this paper was submitted to the IEEE Sensors Journal on $14 / 12 / 2021$ and is currently under review. encodes the depth or distance to the corresponding scene point. The ensemble of these binary sequences leads to sensing matrices, where the arrangement and properties of which are fundamental in CS theory. Good sensing matrices yield low reconstruction errors, and inherently low computational load. The obtainment of such matrices is still an open area of research and debate. In this paper, we focus on two important characteristics of good sensing matrices, such as density and incoherence. The density of a sensing matrix is defined as the ratio between non-zero and total number of elements of the matrix, whereas the incoherence provides an indication of the degree of dissimilarity between the different columns. The former reduces the memory footprint during signal processing. The latter guarantees an efficient scattering of the spatial domain with fewer measurements and reduces the likelihood of failure during reconstruction. Despite classical CS techniques assume no prior knowledge of the signal to retrieve, an insight into the information inherent in the measurements and their relationship with the sensing sequences used for their attainment must not be dismissed. Several researches have been carried out [7], [8] to guide the sensing and recovery processes in a coupled manner. Haupt [9] introduces the concept of Compressive Distilled Sensing (CDS) which consists of the concatenation of sampling and support refinement steps. Malloy [10] develops further these concepts by proposing Compressive Adaptive Search and Sense (CASS) and introduces a number of initial dyadic partitions of the spatial domain and posterior bisections of the most promising subsets. Most of these schemes are based 
on the adaptation of the signal support for each single signal making impractical their use in conventional ToF sensors. In this paper, we firstly propose a conceptually-simple adaptive method which uses an unique illumination system for the entire sensor array, coined Sliced OMP. The spatial domain is initially divided into a number of partitions. A reduced number of initial measurements yields a significant reduction of the signal support by shortlisting a set of different support elements. Then, an effective greedy algorithm, such as OMP, is applied to recover depth and amplitude values. The main advantage of OMP with respect to other sparse recovery approaches is the reduced computational cost associated to signal recovery in highly-sparse scenarios, since it requires $s$ iterations to deliver an $s$-sparse vector, i. e., $s$ targets per observation direction. Moreover, we propose several sensing schemes which guarantee an efficient scattering of the spatial domain and rely on the optimization of coherence properties. Secondly, a methodology is proposed to extend the applicability and facilitate the implementation of APEG [7] with multiaperture systems [11] by performing independent adaptation for the various zones in which the sensor array is divided. The proposed sensing scheme aims to overcome some of the traditional limitations of conventional ToF systems such as the low lateral resolution, the appearance of motion artifacts, and the difficulties associated to uncontrolled environments and poor lighting conditions. We drastically reduce the exposure time and take advantage of mechanical rotation of the camera in order to cover very wide areas and, eventually, employ such a system in fields currently dominated by LIDARs, such as mobile robotics, surface mapping, or autonomous driving.

The rest of the paper is structured as follows. Section II presents a brief outline of research lines on the field of PBToF imaging systems. In Section III, the sensing scheme and recovery strategies are thoroughly described. Section IV presents the results from the numerical simulations performed to evaluate the performance of the various schemes. Finally, Section V summarizes conclusions and prospective research.

\section{RELATED WORK}

Several efforts have been made to enhance the depth resolution and reduce the computational load associated to the signal recovery in Pulse-Based ToF (PB-ToF) imaging systems. In 2007, Laurenzis [12] demonstrated that a 3D scene could be retrieved in a depth of $650 \mathrm{~m}$ to $1550 \mathrm{~m}$ from only three images with an accuracy of $30 \mathrm{~m}$ and $10 \mathrm{~m}$ for black and white targets, respectively, by accounting for the trapezoid-shaped intensity profiles. This result represented a remarkable increase of the depth resolution with respect to existing depth scanning active imaging systems at that time, and enabled superresolution depth mapping at a lower computational cost. Subsequently in 2011 [13], he presented a theoretical model, called image coding, which drastically enlarged the maximum depth range for an arbitrary number of frames used. He gave a precise prediction of the number of permutations that can be used for image coding, and achieved three-dimensional imaging in a range of $460 \mathrm{~m}$ to $1000 \mathrm{~m}$ using a laser pulse width of $300 \mathrm{~ns}$. In 2012, Tsagkatakis [14] introduced the application of CS techniques to Gated Ranged Imaging (GRI) for the recovery of 1-sparse signals from low sampling rates, namely the ratio between the number of frames employed using GRI and the ones from classical range timed imaging. In his seminal study he also accounted for the attenuation of the reflected signals due to the atmosphere as well as for the presence of noise. He obtained some remarkable results through numerical simulations by using OMP with 10 iterations per reconstruction and evaluating two different resolutions, 128 and 256 depth bins, for several sampling rates between 5\% and $49 \%$ and different noise levels. The aforementioned study was extended in 2015 [15], by using non-negative least absolute shrinkage and selection operator (LASSO) [16] and OMP [17], to the retrieval of 2-sparse signals which considered the presence of an additional translucent object in the scene, obtaining exact retrieval from a sampling rate of $\mathrm{r}=10 \%$, by exploiting the prior knowledge of the shape of the cross-correlation function. Another noticeable work in the field of coded ToF cameras was the one performed by Kadambi in 2013 [18] by undertaking sparse deconvolution to reconstruct sequences of Diracs corresponging to multipath returns relying on pulseshaped autocorrelation function. In his work, he was capable of addressing multi-path interference (MPI), recovering the depth of near-transparent surfaces, looking through diffusers, and generating time-profile movies of sweeping light. Moreover, he assessed several modulation codes, and proposed a tailored sparse deconvolution model using a proximity-based approach to matching pursuit. He also described the low-cost camera prototype used to validate his model. In 2019, Tachella et al. [19] applied point cloud denoising tools to single-photon lidar data, reaching reliable 3D image retrieval up to $320 \mathrm{~m}$. An SPAD array of $32 \times 32$ pixels with $100 \mu \mathrm{m}$ pixel pitch was employed for the experiment, although pixel size from modern modules such as the Kinect Azure [20] currently achieves $5 \mu \mathrm{m}$. Some remarkable examples of the use of convolutional neural networks (CNNs) on image processing may be found in Gruber et al. [21], who demonstrated that a low-cost Complementary Metal-Oxide-Semiconductor (CMOS) gated imager may be converted into a dense depth map camera within at least $80 \mathrm{~m}$ range by learning depth from three gated images, and Chen et al. [22] who used signals under very short exposure times via learning process to obtain a depth map from ToF raw measurements. In 2021, A. Schönlieb et al. [23] proposed two novel approaches to mitigate unwanted reflections for under-display ToF imagers, based on the combination of Continuous Wave $(\mathrm{CW})$ measurements with coded modulation, and on measurements with two different frequencies.

\section{ill. Methodology}

\section{A. ToF Sensing Scheme}

The camera rotates in order to cover a very wide area up to, eventually, $360^{\circ}$. The total number of acquisitions per pixel, $N_{\mathrm{t}}$, may be obtained either by acquiring $N_{\mathrm{t}}$ measurements at a number of angular positions with no FOV overlap between them, or by taking one measurement per pose at $N_{\mathrm{t}}$ positions with $F O V_{\mathrm{h}} / N_{\mathrm{t}}$ mismatch between them, being $F O V_{\mathrm{h}}$ the horizontal field-of-view of the camera. The ToF camera setup is shown in Fig. 1. 


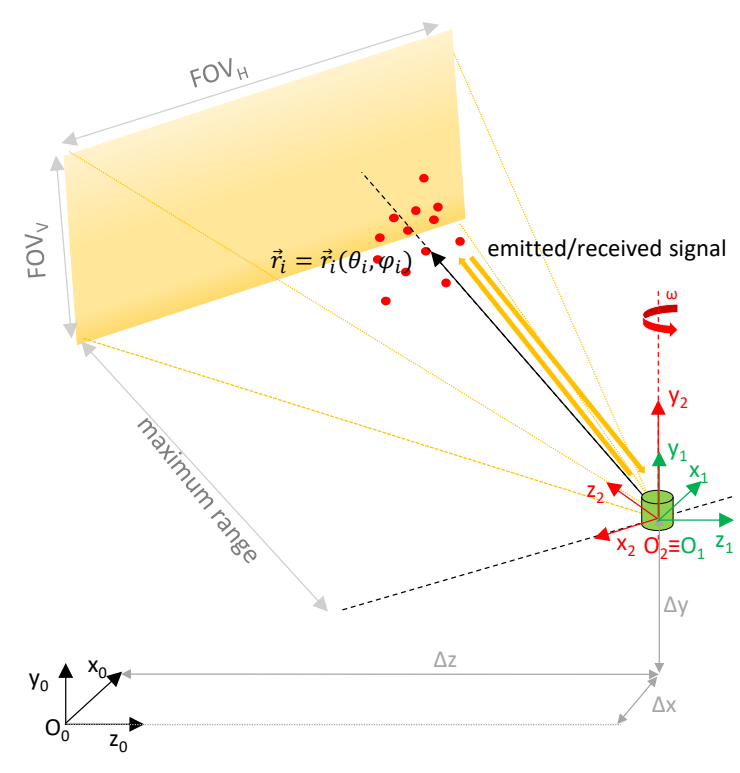

Fig. 1. ToF working principle of the proposed system.

Three different coordinate systems are considered in the camera model. Firstly, the global coordinate reference system, $O_{x_{0} y_{0} z_{0}}$, is the fixed reference for any relative rotation or translation of the camera. Secondly, the camera reference coordinate system, $O_{x_{1} y_{1} z_{1}}$, follows the translation of the camera and maintains its initial orientation. Finally, the camera coordinate system, $O_{x_{2} y_{2} z_{2}}$, follows the translation and rotation of the camera. The camera is initially situated at an arbitrary location with respect to the global coordinate reference system, $\Delta \vec{x}=(\Delta x, \Delta y, \Delta z)$. Any point detected by the camera, $P_{i}$, is given in spherical coordinates by the triplet $\left(\theta_{P_{i}}, \phi_{P_{i}}, r_{P_{i}}\right)$ with respect to the camera coordinate reference system, $O_{x_{1} y_{1} z_{1}}$. The correspondence between uniform sampling on the sensor plane array and the uniform grid on the spherical coordinate system can be assumed due to the large focal distance and the relatively low FOV of the camera.

The emitted periodic signal $p_{\vec{r}_{i}}(t)$, defined for the vector $\vec{r}_{i}=\vec{r}\left(\theta_{i}, \phi_{i}\right)$, along each of the directions considered, interacts with the scene characterized by the scene response function defined in (1), where $\delta(t)$ is the delta function and $\left\{\Gamma_{\vec{r}_{i}}[k], \tau_{\vec{r}_{i}}[k]\right\}_{k=0}^{s-1}$ are the reflectivities and time delays introduced by $s$ return paths of light [24], [25] defined in $t$.

$$
h_{\vec{r}_{i}}\left(t, t^{\prime}\right)=\sum_{k=0}^{s-1} \Gamma_{\vec{r}_{i}}[k] \delta\left(t-t^{\prime}-\tau_{\vec{r}_{i}}[k]\right)
$$

The response function is a shift-invariant function and the interaction with the emitted signal can then be written as the convolution of both signals, (2).

$$
r_{\vec{r}_{i}}(t)=\left(p_{\vec{r}_{i}} * h_{\vec{r}_{i}}\right)(t)
$$

The reflected signal is then correlated at the pixel level, $\left(r_{\vec{r}_{i}} \otimes \Psi_{\vec{r}_{i}}^{j}\right)(t)$, (3) with the controllable shift-invariant response function of the pixel, $\Psi_{\vec{r}_{i}}^{j}(t)$ with $1 \leq j \leq N_{\mathrm{r}}$, which consists of $N_{\mathrm{r}}$ custom binary sequences of $m_{\mathrm{seq}}$ length. In our simulations, each element of the sequence is discretized in $m_{\text {steps }}$ steps, yielding $m_{\text {samples }}=m_{\text {seq }} \times m_{\text {steps }}$, i.e., the number of grid points in which the spatial domain is discretized.

$$
y_{\vec{r}_{i}}^{j}(t)=\left(\left(p_{\vec{r}_{i}} * h_{\vec{r}_{i}}\right) \otimes \Psi_{\vec{r}_{i}}^{j}\right)(t), \quad 1 \leq j \leq N_{r}
$$

Equation (3) may be translated to (4), by making use of cyclic convolution properties [24], since any correlation of energy signals can be rewritten as a convolution, yielding the overall convolution of the three signals in (3). Since the convolutions conmute and, moreover, obey the association law, we may rearrange the products freely, obtaining (4). The interested reader may refer to [24] for more detailed insights into the practical applications of (4) on PB-ToF.

$$
y_{\vec{r}_{i}}^{j}(t)=\left(\left(p_{\vec{r}_{i}} \otimes \Psi_{\vec{r}_{i}}^{j}\right) * h_{\vec{r}_{i}}\right)(t), \quad 1 \leq j \leq N_{r}
$$

As a result, $N_{\mathrm{r}}$ measurements at $t=0$ are produced, yielding a linear system of equations (5), where $\vec{y}_{\vec{r}_{i}}=$ $\left[y_{\vec{r}_{i}}^{j}(t=0)\right]_{i=1}^{N_{\mathrm{r}}}$ is the measurement vector, $A$ is the sensing matrix with $A=\left[\left[a_{j, i}\right]_{j=1}^{N_{\mathrm{r}}}\right]_{i=1}^{m_{\text {samples }}}=\left(p_{\vec{r}_{i}} \otimes \Psi_{\vec{r}_{i}}^{j}\right)\left(t_{i}\right)$, and $\vec{x}_{\vec{r}_{i}}=\left[h_{\vec{r}_{i}}\left(t_{i}\right)\right]_{i=1}^{m_{\text {samples }}}$ is the signal to be reconstructed.

$$
\vec{y}_{\vec{r}_{i}}=A \cdot \vec{x}_{\vec{r}_{i}}
$$

Since $N_{\mathrm{r}} \ll m_{\text {samples }}$ the system is under-determined. Then, we reach a constrained $\ell_{0}$-minimization problem (6), namely, finding the sparsest solution which satisfies (5).

$$
\hat{\vec{x}}_{\vec{r}_{i}}=\underset{\vec{x}_{\vec{r}_{i}}}{\arg \min }|\operatorname{supp}(\vec{x})|, \quad \text { subject to: } \quad \vec{y}_{\vec{r}_{i}}=A \cdot \vec{x}_{\vec{r}_{i}}
$$

Unfortunately, the $\ell_{0}$-minimization problem is non-convex and generally $\mathcal{N} \mathcal{P}$-hard to solve. There are three fundamental approaches to surpass these limitations [26].

- Basis pursuit (BP) is based on the relaxation from $\ell_{0}$ to $\ell_{1}$-minimization and comprises classical algorithms, such as least angle regression (LARS), or LASSO [16], [27][29].

- Greedy algorithms, such as Orthogonal Matching Pursuit (OMP) [17], [25], sequentially retrieve the non-zero coefficients of the signal based on the selection of the support and temporal estimation of the signal.

- Thresholding algorithms, such as Iterative Hard Thresholding (IHT) [30] and Iterative Soft Thresholding [31], [32], keep the largest entries of the signal support selected based on a certain threshold and, then, perform a partial reprojection of the residual.

\section{B. Sparse Signal Recovery Approach}

1) Sliced OMP: In most adaptive sensing methods [7], [9], [10] the support is progressively refined per each single or per small groups of signals, such as groups of adjacent pixels, yielding additional computational load. Moreover, per pixel adaptation is not feasible with current ToF pixel arrays, and adaptation per regions, i. e., groups of pixels, requires making use of multi-aperture arrays [33]. The implementation of these codes as illumination signals would require illumination 
systems capable of independently and simultaneously illuminating different regions of the scene. The method we propose, based on an extension of OMP, provides a cost-effective solution in both, hardware and computational, directions. OMP [17] is a greedy algorithm which aims to obtain the best representation of a signal on an arbitrary redundant basis by projecting the measurement vector $\vec{y}$ and subsequent residuals onto the columns of the sensing matrix. The index of the column which explains best the measurements is added to the temporal support set, i.e., the most correlated column of the sensing matrix with the residual of the measurement vector. In the first iteration of the algorithm, the residual of the measurement vector is initialized as the measurement vector. OMP requires $s$ iterations to deliver a $s$-sparse signal and the exact recovery is guaranteed if the inter-column coherence of the sensing matrix meets the condition $\mu<1 /(2 s-1)$ [34]. A number of simulations were previously performed using random and pseudo-random binary matrices as sensing matrices to validate the applicability of the proposed sensing and recovery strategies [35]. In order to reduce the variability due to random matrix construction, a deterministic approach based on Progressive Edge Growth (PEG) [6], [36] algorithm is proposed for the construction of the sensing matrices. PEG generates Low-Density Parity-Check (LDPC) codes, which locally maximize the girth, i.e., the length of the shortest cycle of the associated Tanner graph or, equivalently, minimize the inter-column coherence of the associated adjacency matrix. Therefore, the good inter-column coherence properties of the adjacency matrices associated to the generated LDPC codes make them good candidates as sensing matrices. However, for a given number of rows $m$, there exists a maximum allowable number of columns which ensures $\mu<1$, a necessary condition to exactly retrieve 1-sparse signals, object of study in our analysis. This number is, at most, the combination without repetition of $N_{\mathrm{deg}}$ non-zero elements, i.e., symbol node degree, in the $m$ rows of the matrix (per column). In our study, the maximum number of columns is defined by the maximum range $\Delta r_{\max }$ and the grid size $\Delta r_{i}$. In order to ensure exact recovery, the spatial domain is sliced in $N_{\mathrm{p}}$ partitions and the sensing matrix is generated as a concatenation of $N_{\mathrm{p}}$ near-to-optimal sensing arrays created via PEG. Previously, a number of acquisitions $\mathrm{N} \leq N_{\mathrm{p}}$ are performed to initially localize the signal within these partitions. Then, OMP is applied over the refined domain. This also allows us to reduce the size of the problem and speed up the recovery process. The proposed approach is illustrated in Fig. 2. Fig. 3 presents the sensing matrices constructed via LDPC-PEG for $N_{\mathrm{r}}=14, m_{\text {samples }}=64$ and $N_{\mathrm{p}}=1,2$, and 4 , and $N_{\mathrm{deg}}=2$, and 3 .

In many scenarios, a sequence of locally optimal decisions may not always yield the globally optimal solution. LDPCPEG sequentially adds $N_{\text {deg }}$ non-zero elements to the columns of the sensing matrix at the locations which optimize the local girth of this column. However, in some cases, such as $N_{\text {deg }}=3$ and $N_{\mathrm{p}}=1$ of Fig. 3 , the coherence does not yield a global minimum. In order to guarantee an optimal inter-column coherence and alleviate the computational cost derived from the construction of LDPC-PEG codes, a second

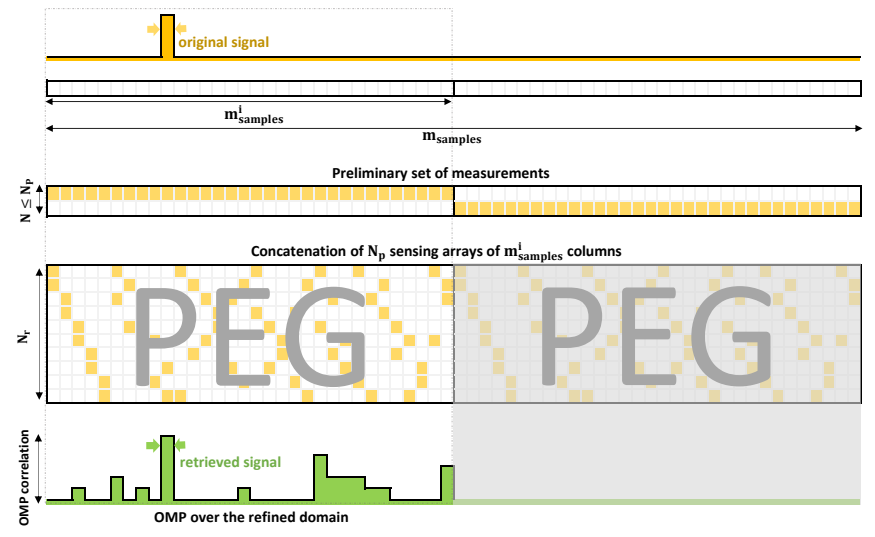

Fig. 2. Proposed approach for signal recovery using Sliced OMP for sparse signal reconstruction and LPDC-PEG for sensing matrix construction.

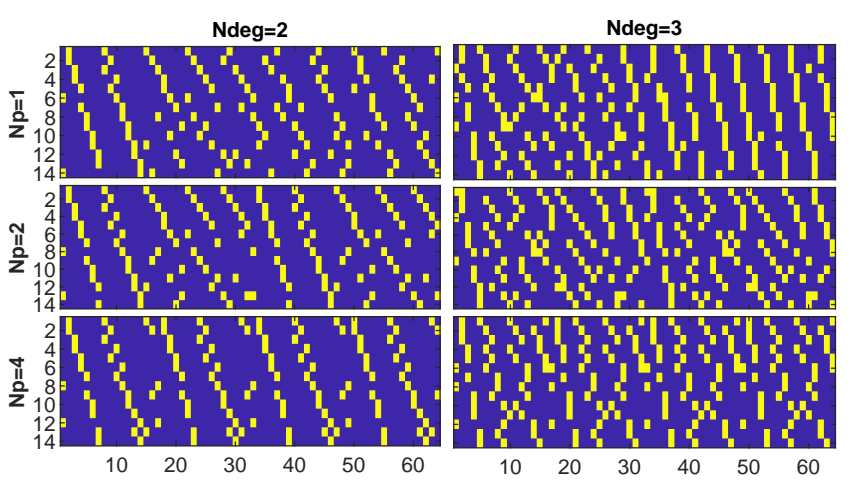

Fig. 3. Sensing matrices constructed via LPDC-PEG.

methodology is proposed to generate the sensing matrices using distinct combinations of $(0,1)$-binary sequences. The algorithm is based on the selection of $m_{\text {samples }}^{\mathrm{i}}$ columns for each partition randomly extracted from all possible combinations without repetition of $N_{\mathrm{deg}}$ non-zero elements in $N_{\mathrm{r}}$ rows, and comprises the following steps:

- $C_{\mathrm{N}_{\mathrm{deg}}}^{N_{\mathrm{r}}}$, the maximum number of possible combinations of $N_{\mathrm{deg}}$ non-zero elements in $N_{\mathrm{r}}$ rows, is calculated (7).

$$
C_{N_{\mathrm{deg}}}^{N_{\mathrm{r}}}=\left(\begin{array}{c}
N_{\mathrm{r}} \\
N_{\mathrm{deg}}
\end{array}\right)=\frac{N_{\mathrm{r}} !}{N_{\mathrm{deg}} !\left(N_{\mathrm{r}}-N_{\mathrm{deg}}\right) !}
$$

- The spatial domain is sliced in a number of partitions $\mathrm{N}_{\mathrm{p}}$ which guarantees a number of columns $m_{\text {samples }^{i}} \leq$ $C_{N_{\text {deg }}}^{N_{r}}$ (8).

$$
N_{\mathrm{p}}=\left\lceil\frac{m_{\text {samples }}}{m_{\text {samples }}^{i}}\right\rceil
$$

- For each partition of the domain, a sensing matrix is constructed concatenating $m_{\text {samples }}^{\mathrm{i}}$ columns randomly selected from $C_{N_{\mathrm{deg}}}^{N_{\mathrm{r}}}$. It should be noted that the size of the last partition may differ from $m_{\text {samples }}^{\mathrm{i}}$ in order to meet the total size of the original spatial domain. 
- $N_{\mathrm{p}}$ sensing arrays are concatenated in order to cover the whole spatial domain.

Fig. 4 presents the sensing matrices constructed via a pure combinatorial approach for $N_{\mathrm{r}}=6,8,10,12$ and $14, \mathrm{~m}=64$ and $N_{\text {deg }}=2$, and 3 .
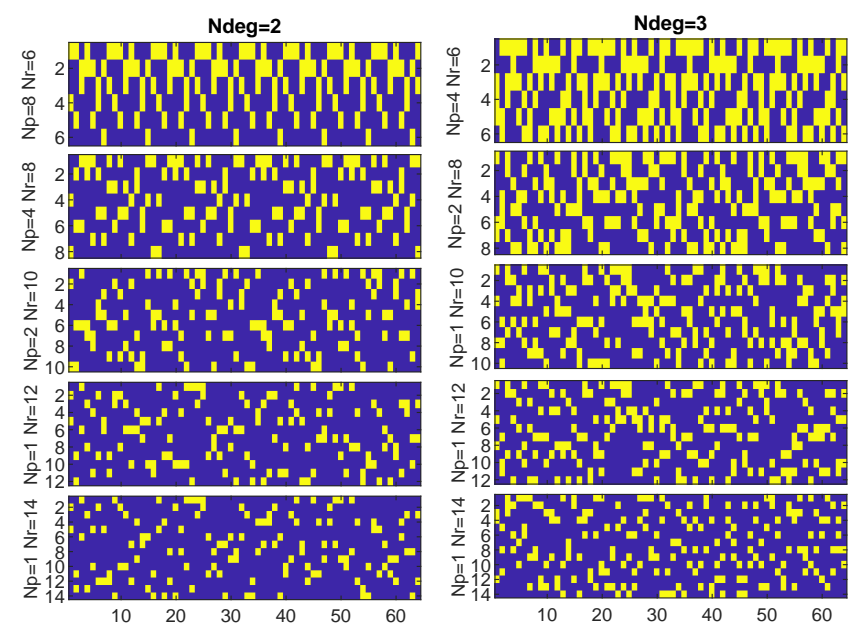

Fig. 4. Sensing matrices constructed via a pure combinatorial approach.
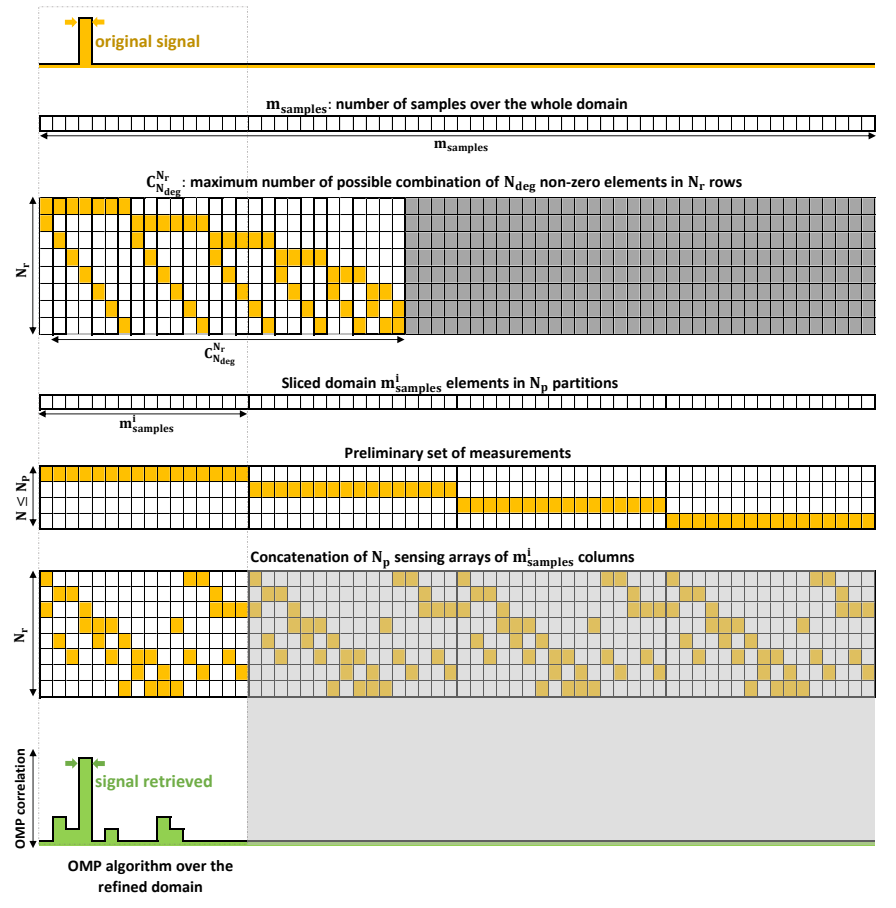

Fig. 5. Proposed approach for signal recovery using Sliced OMP for sparse signal reconstruction and sensing matrix construction using a pure combinatorial approach.

The signal retrieval process consists of an initial localization of the signal within the $N_{\mathrm{p}}$ partitions followed by the application of OMP over the refined domain. The procedure is illustrated in Fig. 5.

This scheme can be generalized for multiple returns per observed direction, i.e., $s$-sparse signals with $s>1$, yielding two possible scenarios. If all the signals fall in the same partition, $s$ iterations of OMP are performed for the refined spatial domain. If at least two sets of signals fall in different partitions, OMP is applied successively to each of the nonempty partitions, and, therefore, the number of measurements required to recover the signals will be directly proportional to the number of non-empty zones.

2) Zoned sparsity-aware APEG: Adaptive Progressive Edge Growth (APEG) [7] is an extension of the PEG algorithm which combines the close-to-optimal properties in terms of inter-column coherence enjoyed by the sensing matrices constructed via PEG-LDPC and the information of the signal support extracted from successive measurements. The algorithm, differently to classical PEG, constructs the sensing matrix per rows by allocating a fixed number of non-zero elements which accounts for the information of the signal support provided by the previous measurements acquired. At the end of the process, the retrieved measurement vector as well as sensing matrix are obtained. In our study, the nature of the signals to be retrieved is very sparse, since in most realistic scenarios we only observe one or two targets per observed direction. Therefore, it seems reasonable to progressively reduce the number of employed non-zero elements, using the adaptive version of the algorithm, as the rows of the measurement matrix are added, by taking into consideration the progressive reduction of the solution space. As most of adaptive methods, the support of the signal in the primary version of APEG is progressively refined per each single signal, i. e., per pixel, making somehow impractical the application of the algorithm to our study, i.e., the fast reconstruction of a large array of 1-sparse signals using relatively simple modulated illumination systems. In order to facilitate the implementation of the algorithm, the identification of the signal support is now performed per group of pixels, the size of which is initially specified. This paves the implementation of such a technique in multi-aperture arrays systems [33].

The proposed approach is illustrated in Fig. 6. Firstly, the sensor array is re-arranged in $N_{\text {zones }}=N_{\mathrm{z}} M_{\mathrm{z}}$, where $M_{\mathrm{z}}, N_{\mathrm{z}}$ are the number of vertical and horizontal divisions performed to the original sensor array. Then, each pixel, $(i, k)$ with $1 \leq k \leq N_{\text {zones }}$ and $1 \leq i \leq N_{\mathrm{k}}, N_{\mathrm{k}}$ representing the number of pixels in each zone, may be defined by the the quadruplet given by its spherical coordinates and intensity values $\left(\theta_{i, k}, \phi_{i, k}, r_{i, k}, \Gamma_{i, k}\right)$ following column-major convention.

The recovery scheme is as follows:

- Preliminary determination of noise threshold for each pixel $\epsilon_{i, k}$ by performing a slicing the spatial domain in $N_{\mathrm{p}}=2$ partitions and acquiring a set of preliminary measurements. The average value of the acquired measurements is conservatively considered as the noise level threshold. If the algorithm is to be generalized to the retrieval of s-sparse signals, with $s>1$, more elaborated discriminant analyses such as the one proposed by Otsu [37] for gray-level histograms may be considered.

- Generation of $N_{\text {zones }}$ sensing matrices $A_{k} \in$ $\mathbb{R}^{N_{\mathrm{r}} \times m_{\text {samples }}}$ and measurement vectors $\vec{y}_{i, k}$ via APEG.

- Implementation of sparse recovery algorithm. OMP is considered for consistency with the rest of the paper. At 


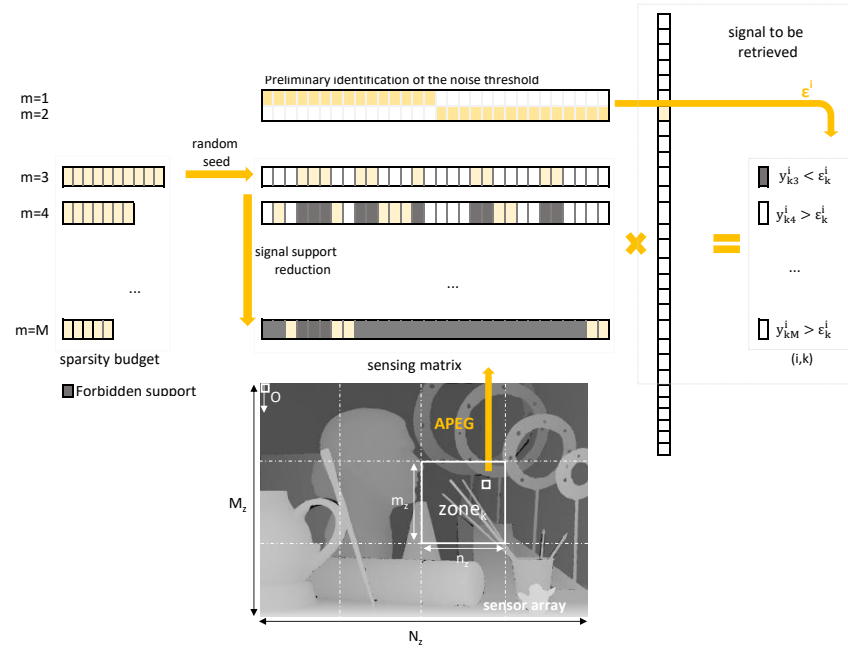

Fig. 6. General proposed approach for signal recovery using Zoned Sparsity-aware APEG.

the end of this step depth and amplitude values $\left(r_{i, k}, \Gamma_{i, k}\right)$ for each pixel are obtained.

The performance gain of Zoned APEG with respect to other sensing methodologies, such as Sliced OMP via LDPC-PEG, is significantly dependant on the geometry and number of array sub-divisions defined a priori and how the various geometries existing in the scene fit into them. The ideal scenario for the implementation of this technique consists of one or several targets within the predetermined partitions, confined in a very narrow spatial range and differentiated from the background. In case of more disperse 3D scenes, where the targets are more coarsely distributed over the spatial domain, the symbol node degree and size of zones must be considerably reduced yielding a worse performance of the algorithm. This opens a window for further research into the initial determination of the geometry and size of zones and how they progressively adapt to the measurements if per-zone programmability of the reference signal for arbitrary geometry zones is possible. A synthetic 3D view is generated consisting of a vertical $2.086 \mathrm{~m} \times 0.700 \mathrm{~m}$ planar object whose center is placed at $z=$ $4 \mathrm{~m}$ with respect to the camera reference system and parallel to the sensor plane. A low-reflectivity background is at a constant distance, $r=8 \mathrm{~m}$, and the maximum range considered is $r_{\max }=10 \mathrm{~m}$. The sensor array is divided in $N_{\text {zones }}=25$. Fig. 7 shows the depth reconstruction error in terms of RMSE, being the first and second sub-indices the corresponding row and column of the $5 \times 5$ divisions the sensor array is divided in, respectively. Several depth values retrieved for different neighboring pixels lie on a narrow range. In these cases, the sensing matrices are progressively adapted to the target location yielding a negligible reconstruction error. In other cases, various distances coexist and the error is significantly higher. The sensing matrices for $N_{\mathrm{r}}=12$ and a reduction of $40 \%$ of the check node degree between measurements are shown in Fig. 8 for the 25 zones the sensor array is divided in. In addition, the histograms of each of the columns of the sensing matrices for each of the zones are presented in Fig. 9, to illustrate the progressive adaptation to the signal support and the adequateness of this approach. The peaks existent in some of the plots mean that the algorithm identifies a tight depth range in which most measurements in the zone are located.

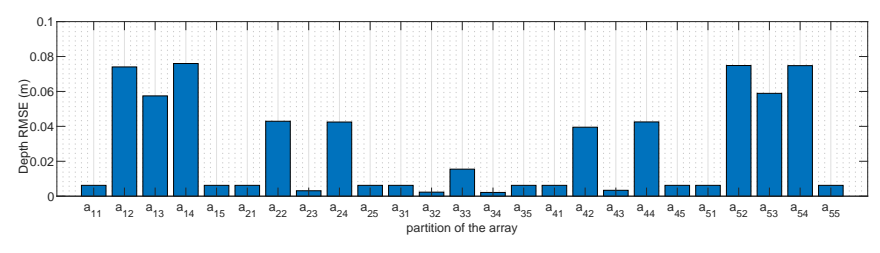

Fig. 7. Depth retrieval error using zoned sparsity-aware APEG with adaptive check node degree.

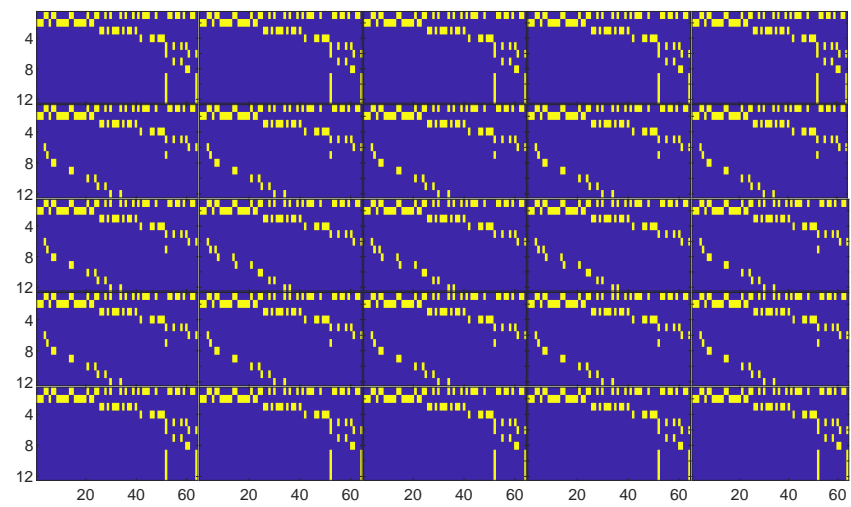

Fig. 8. Sensing matrices for reconstruction via zoned sparsity-aware APEG with adaptive check node degree.

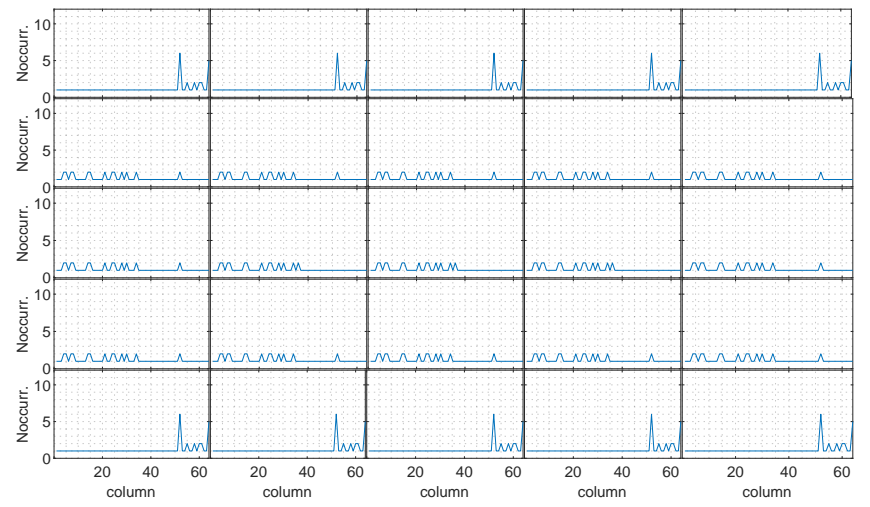

Fig. 9. Columns histogram of sensing matrices via zoned APEG with adaptive check node degree.

\section{Further optimization of ToF sensing schemes accounting for coincidences of raising and falling edges in real operation}

The previous approaches effectively ensure good coherence properties for ideal $(0,1)$-binary sensing matrices. Yet the shifts from one element to another of the sensing matrix do not occur instantaneously and there exist raising and falling times which may deteriorate the coherence properties 
and lead to reconstruction errors. Gupta [38] and GutierrezBarragan [39] proposed a family of novel coding functions based on Hamiltonian cycles on hypercube graphs to avoid the coincidence of raising and falling wavefronts, similarly to Gray codes, and to eventually ensure an optimal coding for ToF imaging. However, Gray codes yield very populated matrices. We propose a different approach by extending the previous schemes and including an additional criterion if a discrimination between the feasible candidate vectors to form the next column can be made during the construction of the sensing matrices taking into consideration the gradient between adjacent columns. Firstly, the absolute difference of the columns with respect to the previous one is calculated. These columns are then concatenated yielding a new matrix of gradients, and the coherence of this matrix is calculated. If the addition of a new column vector from the set of feasible candidate vectors does not lead to unitary coherent, i. e., $\mu<1$, the candidate is considered adequate and is added to the sensing matrix. This step can be applied to both, LDPC-PEG and pure combinatorial, schemes. In the former, it translates into an additional restriction when selecting the last check node for each symbol node from the set of feasible candidates in the Tanner Graph. In the latter, it yields a deterministic arrangement of the sensing matrix rather than a mere random permutation of columns. The procedure is illustrated in Fig. 10 for LDPC-PEG sensing matrix, $N_{\mathrm{p}}=1, N_{\mathrm{r}}=14$, and $N_{\text {deg }}=2$. The ideal matrix presents $\mu=0.5$. However, when applying the instrument response function (IRF), several columns become identical (red boxes in Fig. 10) and this lead to catastrophic failures during recovery, as the matrix of gradients presents $\mu=1$. The IRF was presented in (4) as the cross-correlation of the shift-invariant response function of the pixel and the emitted signal and may be interpreted as the result of applying a low-pass filter to the custom binary sequences generated at the pixel.
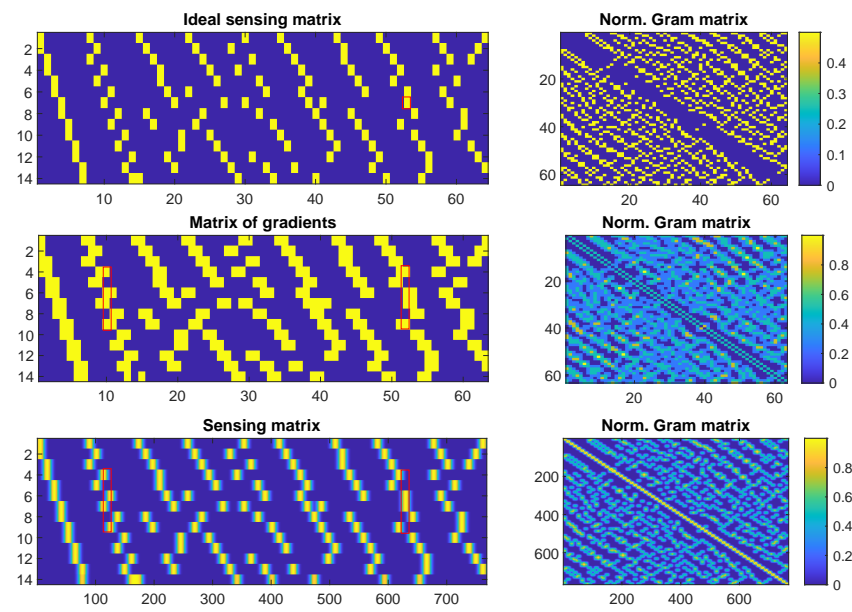

Fig. 10. Sensing matrices via LDPC-PEG and coherence: ideal sensing matrix (first row), matrix of gradients (second row), sensing matrix accounting for IRF (third row).

Fig. 11 and Fig. 12 present the optimized sensing matrices accounting for the coincidence of raising and falling wavefronts, as well as the normalized Gram matrices, which represent a measurement of the similarity between the columns of the sensing matrices.
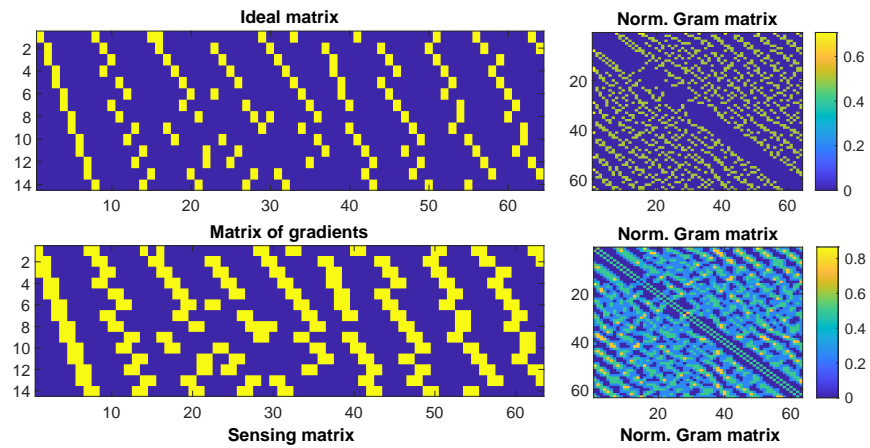

Norm. Gram matrix
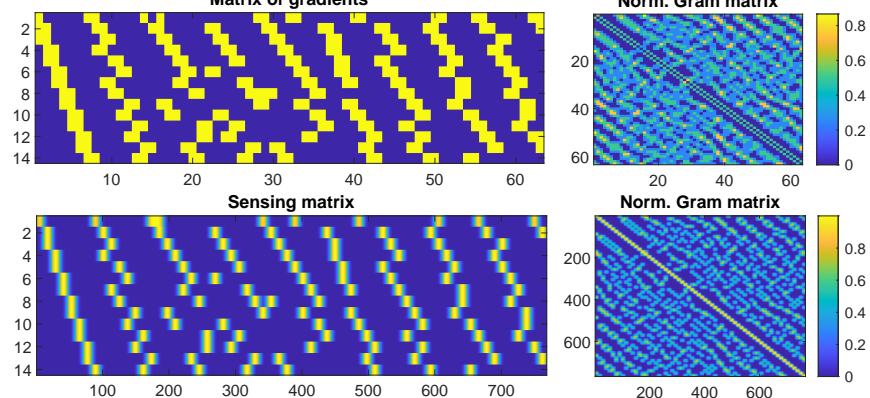

Fig. 11. Optimization of sensing matrices via LDPC-PEG by considering the gradient of adjacent columns in the construction of the sensing matrices and coherence: ideal sensing matrix (first row), matrix of gradients (second row), sensing matrix accounting for IRF (third row).
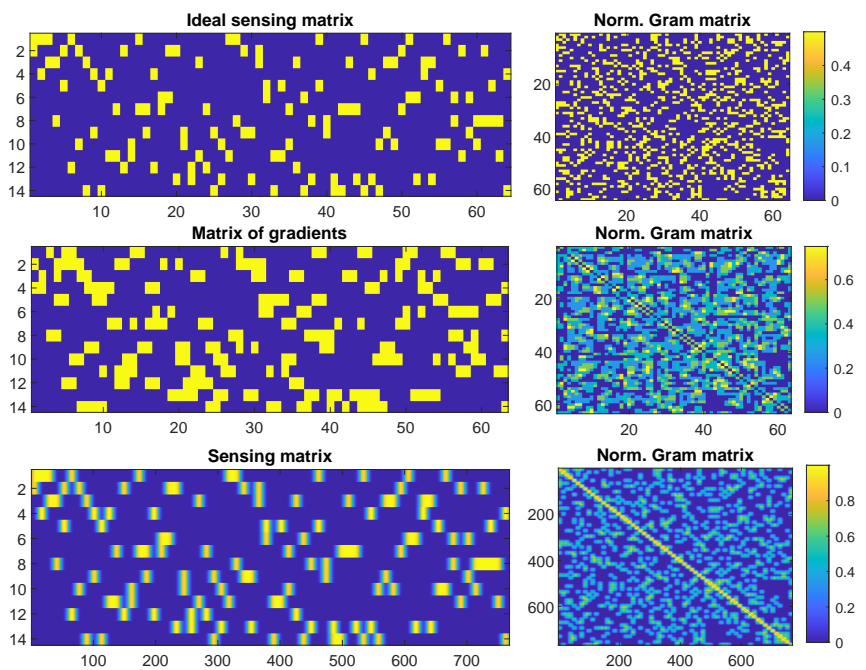

Fig. 12. Optimization of sensing matrices via a pure combinatorial approach by considering the gradient of adjacent columns in the construction of the sensing matrices and coherence: ideal sensing matrix (first row), matrix of gradients (second row), sensing matrix accounting for IRF (third row).

\section{Upper limit for superresolution: IRF and grid size refinement}

In order to obtain a reallistic representation of generated control signals accounting for the IRF, each element of the primary grid is discretized in a number of steps yielding a higher resolution than the size of the original grid. However, there exists an upper limit for this discretization. Beyond this limit, several elements are identical and, therefore, the coherence of the sensing matrix reaches an unitary value, as 

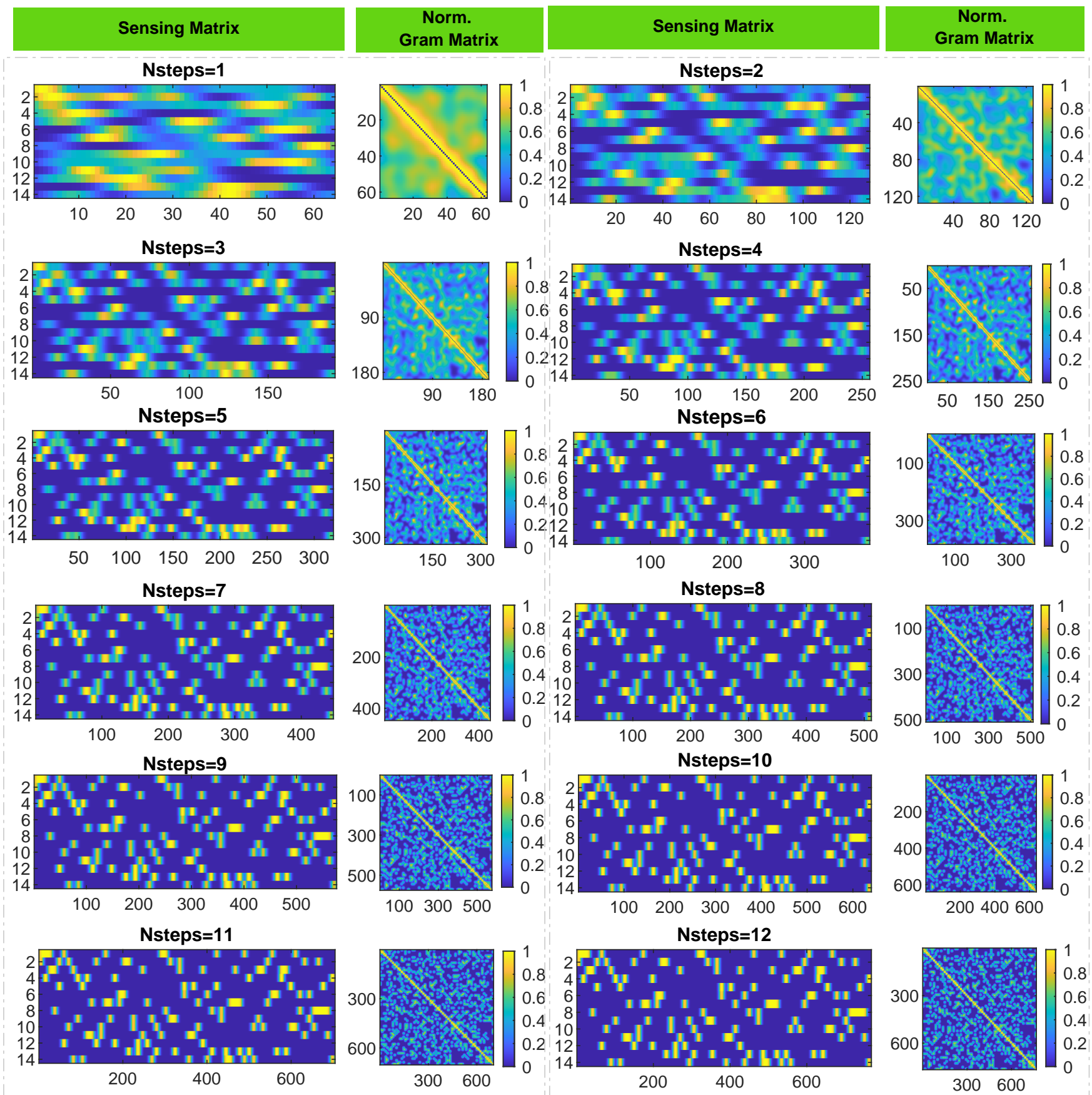

Fig. 13. Upper superresolution limit, maximum grid refinement, for sensing matrices via a combinatorial approach and considering the gradient of adjacent columns.

illustrated in Fig. 13. We observe a concentration of similar values close to the diagonal of the normalized Gram matrix, i.e., there are a few elements near to the center of the binary item in the primary code, which progressively reduce in number but present higher coherence yielding $\mu=1$ for $N_{\text {steps }} \geq 10$, i. e., the upper limit for the grid superresolution and, therefore, beyond this limit the uniqueness of the columns of the sensing matrix cannot be guaranteed.

\section{Numerical Results}

Several simulations are performed using the ground truth (GT) extracted from the disparity and intensity maps of the Middlebury stereo datasets 2003 and 2005 [40]-[42]. $N_{\mathrm{r}}$ sequences of length $m_{\text {seq }}=64$ for a maximum range $\Delta r_{\max }=10 \mathrm{~m}$ are considered. The sequences are discretized in groups of $m_{\text {steps }}=12$. The discretization size is selected beyond the upper superresolution limit in order to avoid any limitation from the grid resolution, as the support index, to 
which a discrete depth is associated, will be selected from the various columns which share identical correlation with the residual of the measurement vector. Then, a Gaussian filter of standard deviation, $\sigma=12 \mathrm{~ns}$, is applied to obtain a realistic representation of the measurement functions. This value corresponds to the real instrument response function (IRF) of a PMD 19k-S3 ToF sensor [24]. The performance of the various sensing schemes is evaluated in terms of depth by presenting the Root Mean Square Error (RMSE) for each dataset [40]-[42]. In addition, the noise present in the measurements is accounted for by adding white Gaussian noise with Signal-to-Noise Ratio (SNR) of SNR $=5 \mathrm{~dB} . N_{\text {real }}=64$ realizations are considered in this analysis. The camera is placed at $\Delta \vec{r}_{0}=(0 \mathrm{~m},-0.1 \mathrm{~m}, 0.2 \mathrm{~m})$ with respect to the global coordinate system, and the measurements are acquired at angular positions with $\Delta \theta=-15 \mathrm{deg}, 15 \mathrm{deg}$. This section is focused on the study of the depth retrieval. Indeed, no significant differences in the recovered amplitude values are found between the cases considered, with $R M S E_{\text {amplitude }}<$ $1 \%$ for $N_{\mathrm{r}}>6$ for all datasets. This is due to the fact that, even in the case of several columns with identical coherence with respect to the measurement vector, OMP yields similar values when performing the pseudo-inverse of the selected column, whether the correct depth is retrieved or not, leading to errors only dependant on the level of added Gaussian noise.

\section{A. Sliced OMP}

Fig. 14 presents the depth reconstruction errors for Sliced OMP using sensing matrices constructed via LDPC-PEG as well as the modified version of LDPC-PEG by considering the matrix of gradients during the selection of candidates for Art and Teddy dataset [40]-[42]. It must be noted the LDPC-PEG in both versions commence with a random selection of $N_{\mathrm{deg}}$ non-zero elements in the first column. We observe that the reconstruction error for classical LDPC-PEG is localized in a very narrow region at which the OMP algorithm catastrophically fails, due to the similarity of columns of the sensing matrix. A more detailed statistical analysis shows that there only exist a reduced number of outliers and that median, 25-percentile and 75-percentile values present exact reconstruction for Art dataset. Indeed, the percentage of exactly recovered targets exceed $96 \%$ for all datasets. This error responds to the collision of raising and falling wavefronts when the IRF and posterior discretization are implemented, as described in previous sections. We find a significant improvement of the recovery algorithm if the additional restriction is imposed to the set of candidates based on the gradient between the consecutive columns. This can be easily observed in cases such as $N_{\mathrm{p}}=1, N_{\mathrm{r}}=14$, and $N_{\mathrm{deg}}=2$ for the dataset Art in which the median depth reconstruction error $R M S E_{\text {depth }}$ decreases from $0.684 \mathrm{~m}$ to $0.001 \mathrm{~m}$ and the average depth reconstruction error $R M S E_{\text {depth }}$ from $0.576 \mathrm{~m}$ to $0.128 \mathrm{~m}$ when the gradient discriminant is considered.

Secondly, Fig. 15 presents the depth reconstruction error for Sliced OMP via a pure combinatorial approach and the extension of the algorithm by including the gradient between adjacent columns. Each new column of the sensing matrix is

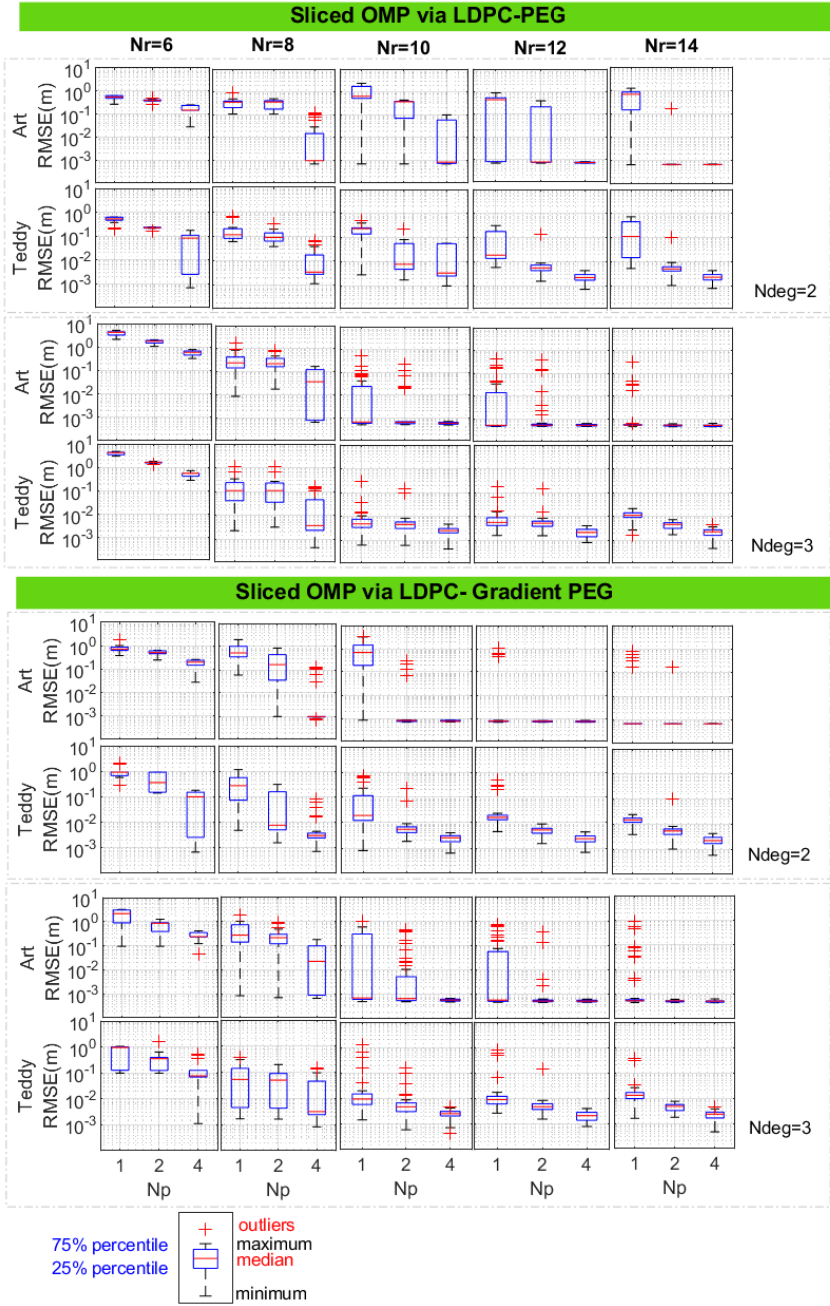

Fig. 14. Depth reconstruction error for sliced OMP via LDPC-PEG.

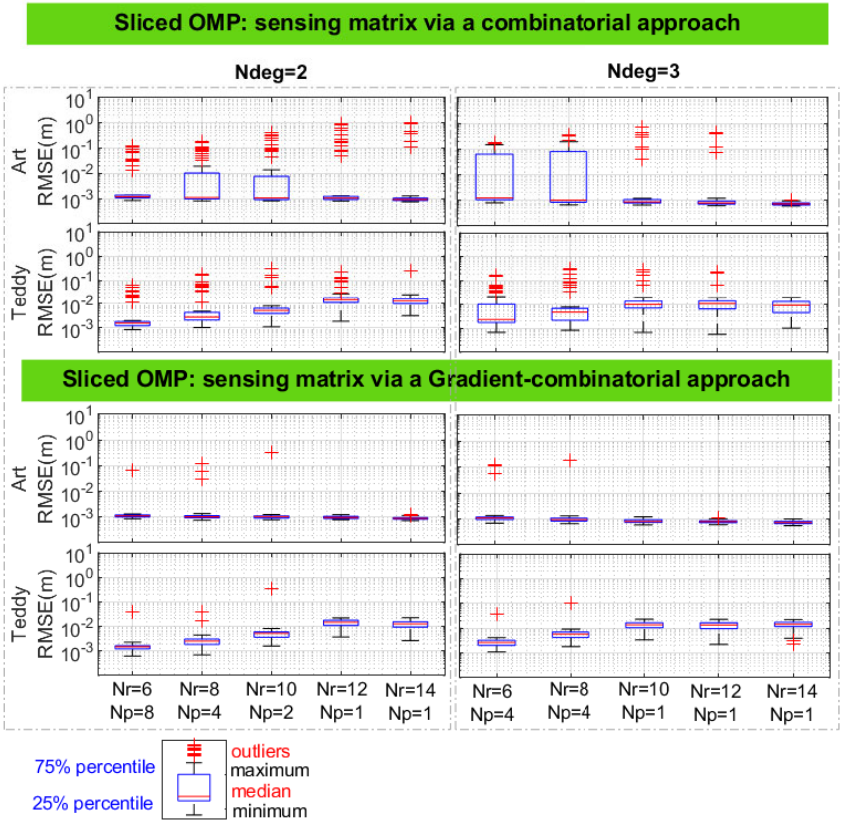

Fig. 15. Depth reconstruction error for sliced OMP via combinatorial approach. 


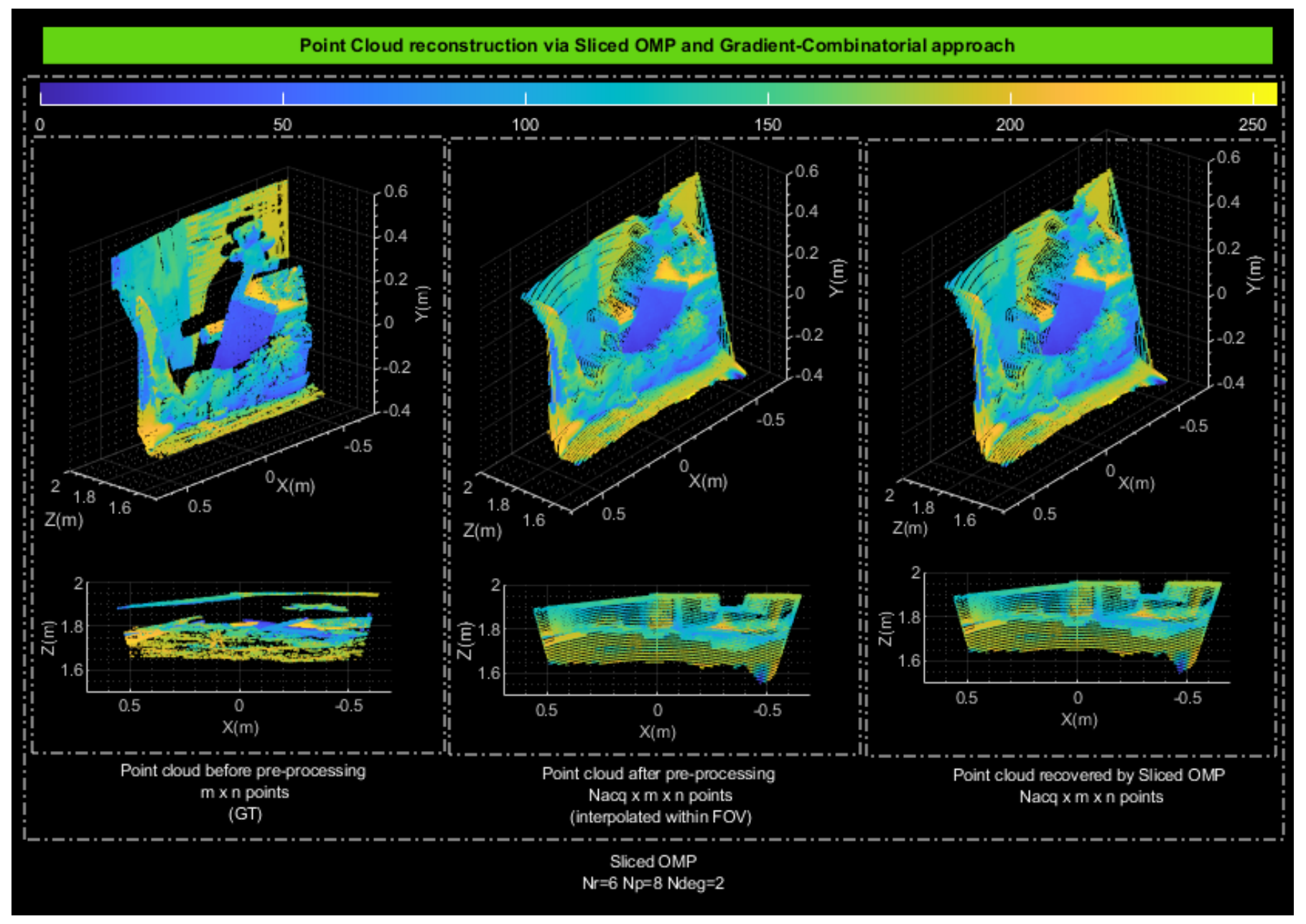

Fig. 16. PCs from Middlebury dataset Teddy: GT (left), PC after pre-procesing (center), and PC retrieved by Sliced OMP via modified version of combinatorial approach.

selected in such a way that this addition optimizes the coherence of the matrix of gradients between adjacent columns. It is observed a good performance of the recovery algorithm with $R M S E_{\text {depth }} \sim \mathcal{O}(\mathrm{mm})$ for a total number of measurements, $N_{\mathrm{t}} \geq 10$, for the modified version of the algorithm. It is also shown a net reduction of the variability of the recovery error when ordering the sensing matrix accounting for the collision of raising and falling wavefronts from consecutive columns.

Fig. 16 presents the point cloud (PC) recovered by Sliced OMP via the extended version of the combinatorial approach for $N_{\mathrm{r}}=6, N_{\mathrm{p}}=8$, and $N_{\mathrm{deg}}=2$. GT from Middlebury dataset Teddy is presented at the left hand side of the figure. The data is then post-processed, as presented in the center of the figure, in order to fit into the viewing angle of the camera, $\theta \in\left(\theta_{1}-F O V_{\mathrm{h}} / 2, \theta_{N_{\mathrm{acq}}}+F O V_{\mathrm{h}} / 2\right)$, being $N_{\text {acq }}$ the number of angular positions considered, and to provide as many points as the number of pixels for each angular position.

\section{B. Sliced OMP for the recovery of multiple returns per observed direction}

The applicability of Sliced OMP is evaluated for multiple returns per observed direction, i.e., 2-sparse signals, using sensing matrices constructed via a combinatorial approach and considering the gradient between adjacent columns as an intermediate verification step. The previous sensing scheme is extended by making use of the preliminary set of measurements to define the boundaries of the refined spatial domain. As discussed in Section III, two different scenarios may be found: If each of the 2 targets fall in a different partition, the problem is equivalent to recover two 1-sparse signals in each of the refined domains and the total number of measurements is $N_{\mathrm{t}}=N_{\mathrm{p}}+2 \times N_{\mathrm{r}}$. Else, if the two targets fall in the same partition, the problem yields two iterations of OMP over the same partition. Since the first scenario is already studied in the previous sections, we focus on the study of the second case, by adding a translucent plane in the view, parallel to the $O x_{1} y_{1}$-plane at a distance $d_{\pi}=0.3 \mathrm{~m}$. Then, Sliced OMP via Gradient-combinatorial approach is applied. Since the output of the algorithm throws $N_{\mathrm{p}}=1$, a $N_{\mathrm{r}} \times m_{\text {samples }}^{i} / N_{\mathrm{p}}^{\prime}$ submatrix is extracted from the matrix generated by the combinatorial approach in order to evaluate the sensitivity of the reconstruction algorithm to the spatial domain refinement, being $N_{\mathrm{p}}^{\prime}=2$. Fig. 18 presents the GT (purple) and the PC after pre-processing (green) at the left hand side of the figure, as well as the GT (purple) and the PC recovered by Sliced OMP via Gradient-combinatorial approach (green) with $N_{\mathrm{r}}=28, N_{\mathrm{p}}=2$, and $N_{\mathrm{deg}}=2$ at 
the right hand side of the figure, for Art dataset plus a low reflectivity translucent plane at $d_{\pi}=0.3 \mathrm{~m}$.

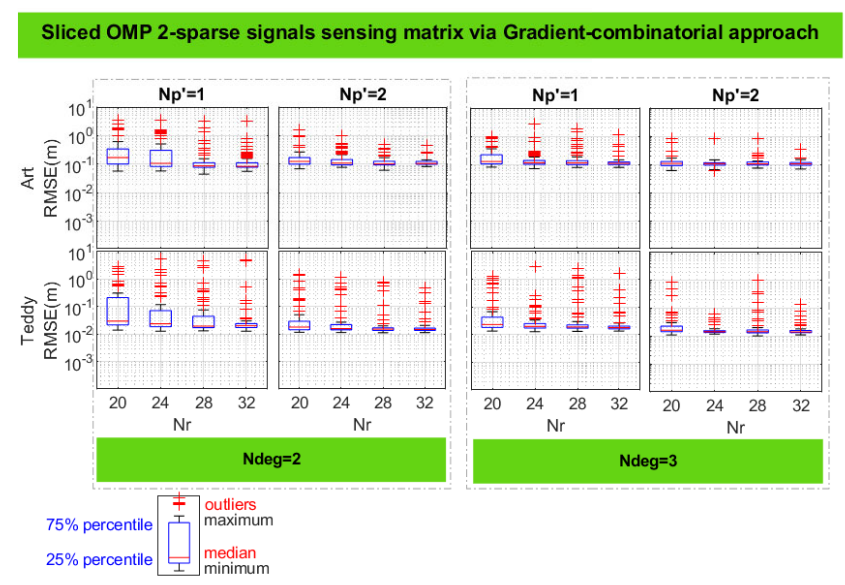

Fig. 17. Depth reconstruction error for Sliced OMP via modified version of pure combinatorial approach for the recovery of 2-sparse signals, by including a translucent plane in the view.

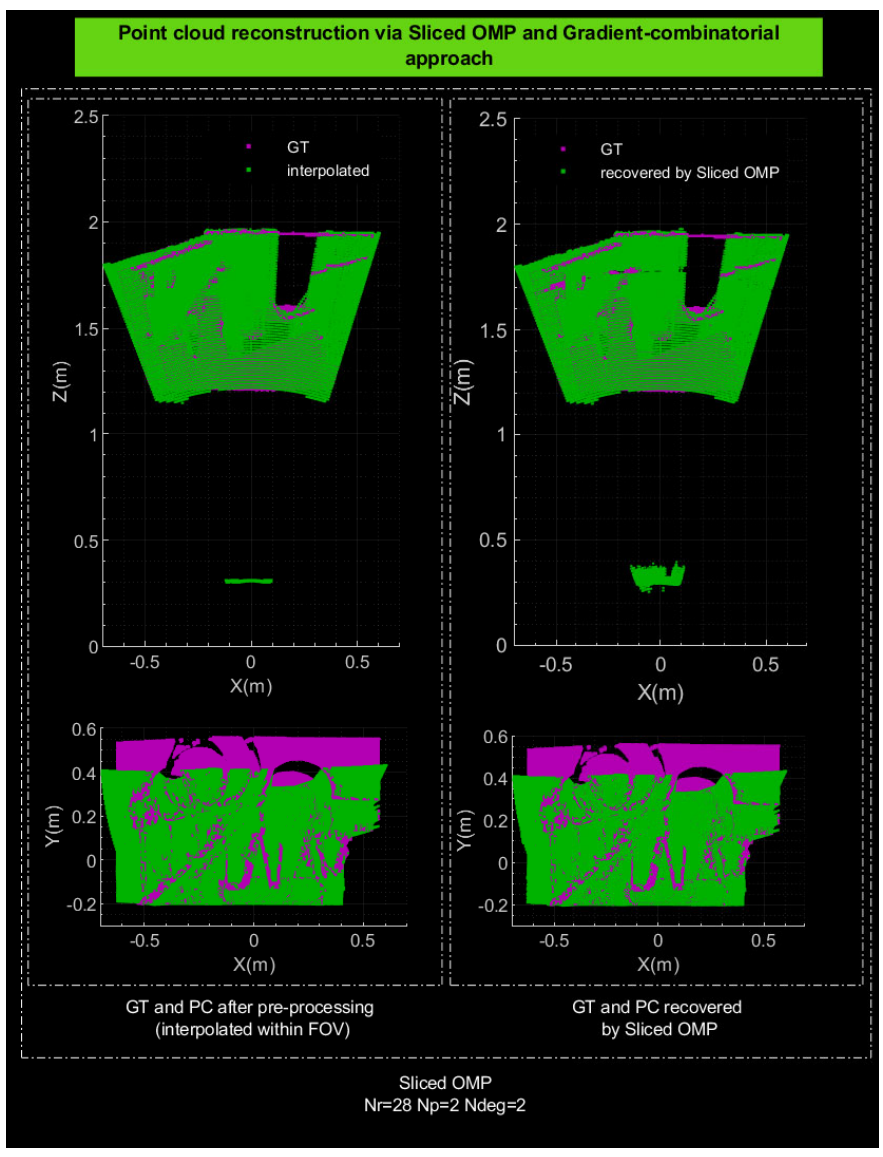

Fig. 18. PCs from Middlebury dataset Art: GT and PC after preprocesing (left). GT and PC retrieved by Sliced OMP via modified version of combinatorial approach (right).
TABLE I

AVERAGE COMPUTATIONAL TIMES PER RETRIEVED SIGNAL FOR OMP AND $\boldsymbol{l}_{1}$-MINIMIZATION LOG-BARRIER ALGORITHMS.

\begin{tabular}{|l|l|l||l|l|}
\hline \multicolumn{3}{|c||}{ Measurements } & \multicolumn{2}{c|}{ Computational times (s) } \\
\hline$N_{\mathrm{t}}$ & $N_{\mathrm{p}}$ & $N_{\mathrm{r}}$ & $\ell_{1}$-min. log-barrier & OMP \\
\hline 14 & 8 & 6 & $7.17 \times 10^{-3}$ & $7.18 \times 10^{-5}$ \\
\hline 12 & 4 & 8 & $1.58 \times 10^{-2}$ & $1.21 \times 10^{-4}$ \\
\hline 12 & 2 & 10 & $3.12 \times 10^{-2}$ & $2.22 \times 10^{-4}$ \\
\hline 12 & 1 & 12 & $5.15 \times 10^{-2}$ & $5.53 \times 10^{-4}$ \\
\hline 14 & 1 & 14 & $7.00 \times 10^{-2}$ & $5.64 \times 10^{-4}$ \\
\hline
\end{tabular}

Precision 3650 MT X2JJF i9-10900K

\section{Sliced OMP Vs $\ell_{1}$-minimization schemes}

The computational time for Sliced OMP with respect to other classical recovery algorithms based on $\ell_{1}$-minimization is the main reason for the selection of such greedy algorithm. This choice is supported by numerical simulations on the Middlebury dataset Teddy [40]-[42], as presented in Table I. The log-barrier algoritmhm from $\ell_{1}$-magic library, [43], plus posterior thresholding of the highest $s$ elements for $s$-parse signals, is selected for this comparison. Since $\ell_{1}$-minimization schemes are based on the implementation of a linear solver, the uniqueness of the columns of the sensing matrix, and, therefore, the grid resolution is limited to $n_{\text {steps }}=8$, well below the upper superresolution limit of the grid. As expected, it is found a significant increase of computational time per recovered signal, up to two orders of magnitude, when using a $\ell_{1}$-minimization scheme, making somehow impractical the sequential implementation of such algorithms for all pixels in the array in our sensing scheme for real-time applications.

\section{Zoned sparsity-aware APEG}

The depth and intensity maps [40]-[42] are now divided in $N_{\text {zones }}$ zones, and then the Zoned APEG algorithm is applied in parallel to each of them. Then, OMP is applied to recover depth and amplitude for each pixel. The depth retrieval errors are presented in Fig. 19. The check node degree is progressively reduced from one set of measurements to another with a rate $=60 \%$. The division of the array into various zones does not provide a significant gain of performance with respect to the the original array with depth errors $R M S E_{\text {depth }} \sim$ $\mathcal{O}(\mathrm{cm})$ for $N_{\mathrm{r}} \geq 12$. We argue the algorithm may be significantly optimized if the geometry and size of the initial partitions of the array can progressively be readjusted to the information acquitted from the scene under study. However, this is not possible, since all commercial ToF cameras use a single control signal that is common for all pixels. In the case of having multi-aperture arrays, each aperture is restricted to a rectangular fixed region. Enabling this capacity would mean a number of control lines equal to the number of desired regions would have to be wired to each pixel. Then, depending on to which region should belong, the pixel should be driven by one line or another. 


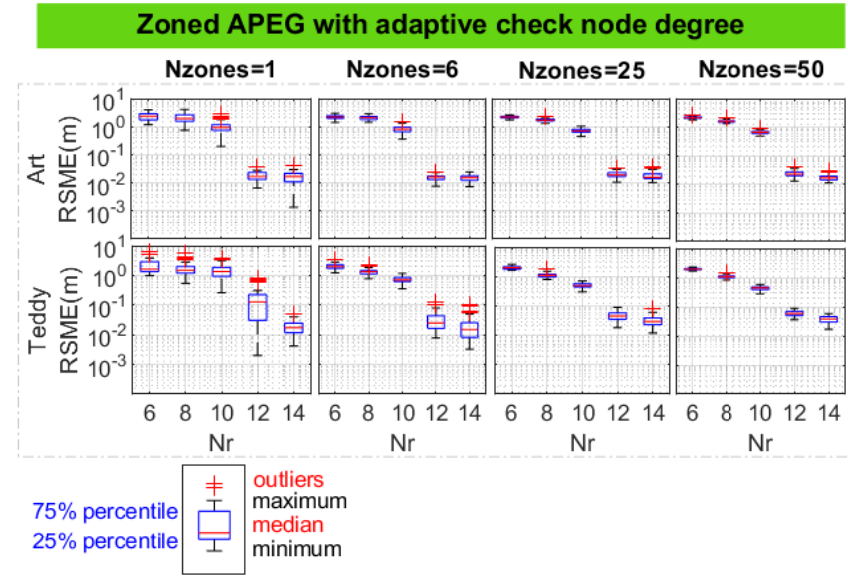

Fig. 19. Depth reconstruction error for Zoned APEG.

\section{Conclusions}

In this paper, we have proposed two practical schemes to reconstruct very-wide, long-range and highly-sparse scenarios with few measurements, low computational load, and relatively simple illumination systems. Thorough simulations have demonstrated the validity of the considered assumptions and pave the way to implement such systems in the ToF camera prototype currently being developed at ZESS. We have focused on the procurement of good sensing matrices by guaranteeing an efficient scattering of the spatial domain, exploring and comparing various construction methodologies to minimize the number of required measurements and to enable the performance in nearly real-time operation. The results show good performances for the deterministic techniques, i.e., LDPCPEG and pure combinatorial approaches. It is noted that the latter presents a better precision for a given measurement budget, since it is shown to yield always the optimal solution in terms of inter-column incoherence.

Also, we have optimized both schemes by introducing a second criterion in the selection of the non-zero elements in the sensing matrices in order to avoid coincidence of raising and falling wavefronts from the various custom sequences, when IRF is applied. We have calculated the upper superresolution limit, i. e., the minimum reachable grid size that still ensures $\mu<1$. Additionally, we have demonstrated that the recovery of the signals may be accomplished with high probability in all cases by building upon a conceptually simple, fast and efficient greedy algorithm, such as OMP, which presents computational times up to two orders of magnitude lower than other approaches based on the minimization of the $\ell_{1}$-norm. Moreover, we have generalized the proposed methodology to address the recovery of $s$-sparse signals, being $s>1$ the number of returns per observed direction. Finally, we have outlined a way of extending the applicability of an existing adaptive CS algorithm, such as APEG, to more practical illumination systems. We have shown the effectiveness of this sensing scheme in scenarios in which the scene objects are severely concentrated in narrow regions of the space.

\section{REFERENCES}

[1] E. J. Candes and M. B. Wakin, "An introduction to compressive sampling," IEEE Signal Processing Magazine, vol. 25, no. 2, pp. 21-30, 2008.

[2] E. Candes, J. Romberg, and T. Tao, "Robust uncertainty principles: exact signal reconstruction from highly incomplete frequency information," IEEE Transactions on Information Theory, vol. 52, no. 2, pp. 489-509, 2006.

[3] E. J. Candes and T. Tao, "Near-optimal signal recovery from random projections: Universal encoding strategies?" IEEE Transactions on Information Theory, vol. 52, no. 12, pp. 5406-5425, 2006.

[4] D. L. Donoho, "Compressed sensing," IEEE Transactions on Information Theory, vol. 52, no. 4, pp. 1289-1306, 2006.

[5] L. Gan, T. T. Do, and T. D. Tran, "Fast compressive imaging using scrambled block hadamard ensemble," in 2008 16th European Signal Processing Conference, 2008, pp. 1-5.

[6] X.-Y. Hu, E. Eleftheriou, and D.-M. Arnold, "Progressive edge-growth tanner graphs," in GLOBECOM'01. IEEE Global Telecommunications Conference (Cat. No.01CH37270), vol. 2, 2001, pp. 995-1001 vol.2.

[7] M. Heredia Conde, K. Hartmann, and O. Loffeld, "Simple adaptive progressive edge-growth construction of LDPC codes for close(r)-tooptimal sensing in pulsed ToF," in 2016 4th International Workshop on Compressed Sensing Theory and its Applications to Radar, Sonar and Remote Sensing (CoSeRa), 2016, pp. 80-84.

[8] S. Ji, Y. Xue, and L. Carin, "Bayesian compressive sensing," IEEE Transactions on Signal Processing, vol. 56, no. 6, pp. 2346-2356, June 2008.

[9] J. D. Haupt, R. G. Baraniuk, R. M. Castro, and R. D. Nowak, "Compressive distilled sensing: Sparse recovery using adaptivity in compressive measurements," in 2009 Conference Record of the Forty-Third Asilomar Conference on Signals, Systems and Computers, 2009, pp. 1551-1555.

[10] M. L. Malloy and R. D. Nowak, "Near-optimal adaptive compressed sensing," IEEE Transactions on Information Theory, vol. 60, no. 7, pp. 4001-4012, 2014.

[11] M. Heredia Conde, K.Kagawa, T. Kokado, S. Kawahito, and O. Loffeld, "Single-shot real-time multiple-path time-of-flight depth imaging for multi-aperture and macro-pixel sensors," in ICASSP 2020 - 2020 IEEE International Conference on Acoustics, Speech and Signal Processing (ICASSP), 2020, pp. 1469-1473.

[12] M. Laurenzis, F. Christnacher, and D. Monnin, "Long-range threedimensional active imaging with superresolution depth mapping," Optics letters, vol. 32, pp. 3146-8, 112007.

[13] M. Laurenzis and E. Bacher, "Image coding for three-dimensional rangegated imaging," Applied optics, vol. 50, pp. 3824-8, 072011.

[14] G. Tsagkatakis, A. Woiselle, G. Tzagkarakis, M. Bousquet, J.-L. Starck, and P. Tsakalides, "Active range imaging via random gating," Proceedings of SPIE - The International Society for Optical Engineering, vol. 8542, 092012.

[15] G. Tsagkatakis, A. Woiselle, G. Tzagkarakis, M. Bousquet, J. Starck, and P. Tsakalides, "Multireturn compressed gated range imaging," Optical Engineering, vol. 54, no. 3, pp. 1 - 12, 2015. [Online]. Available: https://doi.org/10.1117/1.OE.54.3.031106

[16] H. Zou, "The adaptive LASSO ad its oracle properties," Journal of the American Statistical Association, vol. 101, pp. 1418-1429, 022006.

[17] Y. C. Pati, R. Rezaiifar, and P. S. Krishnaprasad, "Orthogonal matching pursuit: recursive function approximation with applications to wavelet decomposition," in Proceedings of 27th Asilomar Conference on Signals, Systems and Computers, 1993, pp. 40-44 vol.1.

[18] A. Kadambi, R. Whyte, A. Bhandari, L. Streeter, C. Barsi, A. Dorrington, and R. Raskar, "Coded time of flight cameras: Sparse deconvolution to address multipath interference and recover time profiles," $A C M$ Transactions on Graphics (TOG), vol. 32, 112013.

[19] J. Tachella, Y. Altmann, N. Mellado, A. McCarthy, R. Tobin, G. S. Buller, J. Tourener, and S. McLaughlin, "Real-time 3D reconstruction from single-photon lidar data using plug-and-play point cloud denoisers," Nature Communications, vol. 10, no. 4984, 2019.

[20] C. S. Bamji, S. Mehta, B. Thompson, T. Elkhatib, S. Wurster, O. Akkaya, A. Payne, J. Godbaz, M. Fenton, V. Rajasekaran, L. Prather, S. Nagaraja, V. Mogallapu, D. Snow, R. McCauley, M. Mukadam, I. Agi, S. McCarthy, Z. Xu, T. Perry, W. Qian, V. Chan, P. Adepu, G. Ali, M. Ahmed, A. Mukherjee, S. Nayak, D. Gampell, S. Acharya, L. Kordus, and P. O'Connor, "IMpixel 65nm BSI 320 mhz demodulated TOF image sensor with $3 \mu \mathrm{m}$ global shutter pixels and analog binning," in 2018 IEEE International Solid - State Circuits Conference - (ISSCC), 2018, pp. 94-96. 
[21] T. Gruber, F. Julca-Aguilar, M. Bijelic, and F. Heide, "Gated2depth: Real-time dense lidar from gated images," in 2019 IEEE/CVF International Conference on Computer Vision (ICCV), 2019, pp. 1506-1516.

[22] Y. Chen, J. Ren, X. Cheng, K. Qian, L. Wang, and J. Gu, "Very power efficient neural time-of-flight," in 2020 IEEE Winter Conference on Applications of Computer Vision (WACV), 2020, pp. 2246-2255.

[23] A. Schonlieb, H. Plank, C. Steger, and N. Druml, "Stray-light mitigation for under-display time-of-flight imagers," IEEE Sensors Journal, vol. PP, pp. 1-1, 112021.

[24] A. Bhandari, M. Heredia Conde, and O. Loffeld, "One-bit time-resolved imaging," IEEE Transactions on Pattern Analysis and Machine Intelligence, vol. 42, no. 7, pp. 1630-1641, 2020.

[25] M. Heredia Conde, Compressive Sensing for the Photonic Mixer Device - Fundamentals, Methods and Results. Springer, 2017.

[26] S. Foucart and H. Rauhut, A Mathematical Introduction to Compressive Sensing. Birkhäuser Basel, 2013.

[27] R. Tibshirani, "Regression shrinkage and selection via the Lasso," Journal of the Royal Statistical Society: Series B (Methodological), vol. 58, pp. 267-288, 011996.

[28] D. Luengo, J. Vía, and T. Trigano, "Efficient iteratively reweighted lasso algorithm for cross-products penalized sparse solutions," in 2020 28th European Signal Processing Conference (EUSIPCO), 2021, pp. 20452049.

[29] O. Loffeld, T. Espeter, and M. Heredia Conde, "From weighted least squares estimation to sparse CS reconstruction," in 2015 3rd International Workshop on Compressed Sensing Theory and its Applications to Radar, Sonar and Remote Sensing (CoSeRa), 2015, pp. 149-153.

[30] T. Blumensath and M. E. Davies, "Iterative hard thresholding for compressed sensing," Applied and Computational Harmonic Analysis, vol. 27, no. 3, pp. 265-274, 2009. [Online]. Available: https://www.sciencedirect.com/science/article/pii/S1063520309000384

[31] $\mathrm{H}$. Bi and G. Bi, "Performance analysis of iterative soft thresholding algorithm for $l_{1}$ regularization based sparse sar imaging," in 2019 IEEE Radar Conference (RadarConf), 2019, pp. 1-6.

[32] Y. Jiao, B. Jin, and X. Lu, "Iterative soft/hard thresholding with homotopy continuation for sparse recovery," IEEE Signal Processing Letters, vol. 24, no. 6, pp. 784-788, 2017.

[33] M. Heredia Conde, K. Kagawa, T. Kokado, S. Kawahito, and O. Loffeld, "Single-shot real-time multiple-path time-of-flight depth imaging for multi-aperture and macro-pixel sensors," in ICASSP 2020 - 2020 IEEE International Conference on Acoustics, Speech and Signal Processing (ICASSP), 2020, pp. 1469-1473.

[34] J. Tropp, "Greed is good: algorithmic results for sparse approximation," IEEE Transactions on Information Theory, vol. 50, no. 10, pp. 22312242, 2004.

[35] A. Lopez Paredes, M. Heredia Conde, and O. Loffeld, "Effective verywide-area 3D ToF sensing," in SENSORS 2021, 2021.

[36] G. Srirutchataboon, A. Bajpai, L. Wuttisittikulkij, and P. Kovintavewat, "Peg-like algorithm for ldpc codes," in 2014 International Electrical Engineering Congress (iEECON), 2014, pp. 1-4.

[37] N. Otsu, "A threshold selection method from gray-level histograms," IEEE Transactions on Systems, Man, and Cybernetics, vol. 9, no. 1, pp. 62-66, 1979.

[38] M. Gupta, A. Velten, S. K. Nayar, and E. Breitbach, "What are optimal coding functions for time-of-flight imaging?" $A C M$ Trans. Graph., vol. 37, no. 2, Feb. 2018. [Online]. Available: https://doi.org/10.1145/3152155

[39] F. Gutierrez-Barragan, S. A. Reza, A. Velten, and M. Gupta, "Practical coding function design for time-of-flight imaging," in IEEE Conference on Computer Vision and Pattern Recognition, CVPR 2019, Long Beach, CA, USA, June 16-20, 2019. Computer Vision Foundation / IEEE, 2019, pp. 1566-1574. [Online]. Available: http://openaccess.thecvf.com/content_CVPR_2019/html/GutierrezBarragan_Practical_Coding_Function_Design_for_Time-OfFlight_Imaging_CVPR_2019_paper.html

[40] H. Hirschmuller and D. Scharstein, "Evaluation of cost functions for stereo matching," in 2007 IEEE Conference on Computer Vision and Pattern Recognition, 2007, pp. 1-8.

[41] D. Scharstein and C. Pal, "Learning conditional random fields for stereo," in 2007 IEEE Conference on Computer Vision and Pattern Recognition, 2007, pp. 1-8.

[42] D. Scharstein and R. Szeliski, "High-accuracy stereo depth maps using structured light," in 2003 IEEE Computer Society Conference on Computer Vision and Pattern Recognition, 2003. Proceedings., vol. 1, 2003, pp. I-I.

[43] E. J. Candes and J. Romberg, " $\ell_{1}$-magic: Recovery of sparse signals via convex programming," 012005 , pp. 1-19.

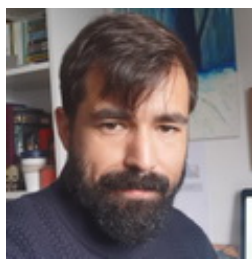

Alvaro Lopez Paredes Alvaro Lopez Paredes received his MEng in Aerospace Engineering by the Technical University of Madrid (UPM) in 2012. In addition, he completed a Master's Degree in Numerical Simulation majoring in Solid Mechanics in 2016, and a Specialist's Degree in Numerical Simulation majoring in Computational Fluid Dynamics in 2020, both from the UPM in collaboration with ANSYS. Alvaro Lopez Paredes was one of the recipients of the 2005 Academic Excellence Prices, awarded by the Government of Madrid, Spain. In 2017, he was selected by the Unit of Excellence Maria de Maeztu at the Department of Astrophysics of Particles of CIEMAT, Spain, and participated in the final engineering phase of the LST-1 Camera protoype for the CTA Project in the Roque de los Muchachos Observatory in La Palma, Spain. After a relevant professional experience in the Oil and Gas industry in France and the United Kingdom, he joined the Marie Skłodowska-Curie Innovative Training Network Project "MENELAOS NT" as an Early-Stage Researcher at the Center for Sensor Systems (ZESS) of the University of Siegen, Germany, where he conducts his PhD studies in the field of very-wide area 3D ToF imaging systems by making use of adaptive Compressive Sensing and sparsity-awareness techniques.

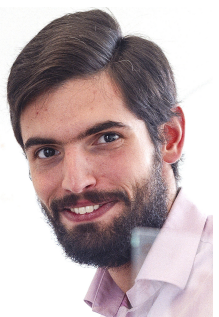

Miguel Heredia Conde (M'14) received the Dr. Eng. degree in the field of sensor signal processing from the University of Siegen, Siegen, Germany, in 2016. In 2013, he joined the Center for Sensorsystems (ZESS), at the University of Siegen. Since then he has also been a member of the Research Training Group GRK 1564 "Imaging New Modalities". Since 2016 he is the Leader of the research group "Compressive Sensing for the Photonic Mixer Device" and since 2020 also the General Manager of the H2020-MSCA-ITN "MENELAOSNT". His current research interests include Time-of-Flight Imaging systems, such as those based on the Photonic Mixer Device (PMD), Compressive Sensing, Computational Imaging, and unconventional sensing. Miguel Heredia was one of the recipients of the 2006 Academic Excellence Prices, awarded by the Government of Galicia, Spain. In 2017 he was awarded the University of Siegen Prize for International Young Academics, for the excellent performance in his doctoral studies. He is a member of the ITG/VDE and the IEEE/SPS.

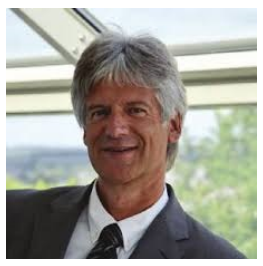

Otmar Loffeld (M'05-SM'06) received the Dr. Eng. degree and the "Habilitation" in the field of digital signal processing and estimation theory in 1986 and 1989, respectively, both from the University of Siegen. Since 1991, he is a professor at the University of Siegen. He has authored two textbooks on estimation theory. In 1995, he joined the Center for Sensorsystems (ZESS) at the University of Siegen and became the chair in 2005. In 2002, he founded the International Postgraduate Program (IPP) "MultiSensorics," and in 2008 established the "NRW Research School on Multi Modal Sensor Systems for Environmental Exploration and Safety (MOSES)" at the University of Siegen. His current research interests include multisensor-data fusion, Kalman filtering techniques for data fusion, optimal filtering and process identification, SAR processing and simulation, SAR interferometry, phase unwrapping, baseline estimation and, recently, bistatic SAR processing. He is a member of the ITG/VDE and Senior Member of the IEEE/GRSS 